\title{
Infant Nutrition
}

National Cancer Institute

\section{Source}

National Cancer Institute. Infant Nutrition. NCI Thesaurus. Code C15264.

Nutritional studies with a focus on the specific nutritional needs of children under the age of 2 . 